\title{
Efecto de recubrimientos comestibles de Aloe vera y alginato de sodio sobre la calidad poscosecha de fresa
}

\section{Effect of Aloe vera and sodium alginate edible coatings on postharvest quality of strawberry}

\author{
Alexis García-Figueroa'; Alfredo Ayala-Aponte²; Martha Isabel Sánchez-Tamayo³
}

\begin{abstract}
${ }^{1}$ Ing. de Alimentos, Aspirante a M.Sc. Universidad del Valle, Facultad de ingeniería. Santiago de Cali - Valle del Cauca, Colombia; e-mail: alexis.garcia@ correounivalle.edu.co; Dhttps://orcid.org/0000-0001-5036-2307

${ }^{2}$ Ing. Agrícola, Ph.D. Universidad del Valle, Facultad de Ingeniería, Escuela de Ingeniería de Alimentos. Santiago de Cali - Valle del Cauca, Colombia; e-mail: alfredo.ayala@correounivalle.edu.co; (Dhttps://orcid.org/0000-0003-0310-3577

${ }^{3}$ Ing. Alimentos, M.Sc. Ph.D.(c). Universidad del Valle, Facultad de Ingeniería. Santiago de Cali - Valle del Cauca, Colombia; e-mail: martha.isabel.sanchez@ correounivalle.edu.co; (Dhttps://orcid.org/0000-0002-8381-9171
\end{abstract}

Cómo citar: García-Figueroa, A.G.; Ayala-Aponte, A.; Sánchez-Tamayo, M.I. 2019. Efecto de recubrimientos comestibles de Aloe vera y alginato de sodio sobre la calidad poscosecha de fresa. Rev. U.D.C.A Act. \& Div. Cient. 22(2):e1320. http://doi.org/10.31910/rudca. v22.n2.2019.1320

Artículo de acceso abierto publicado por Revista U.D.C.A Actualidad \& Divulgación Científica, bajo una licencia Creative Commons CC BY-NC 4.0

Recibido: Mayo 24 de 2018

Aceptado: Junio 26 de 2019

Editado por: Ingeborg Zenner de Polanía

\section{RESUMEN}

Las fresas son frutas altamente perecederas, susceptibles a daño mecánico, deterioro y desórdenes fisiológicos durante su almacenamiento. La aplicación de recubrimientos, se presenta como una alternativa promisoria de tratamiento poscosecha, que permite mantener la calidad de las fresas. El objetivo de esta investigación fue estudiar el efecto de recubrimientos comestibles de sábila ( $A$. vera) y alginato de sodio sobre parámetros de calidad de fresas, durante el almacenamiento refrigerado. Se evaluaron mezclas de recubrimientos comestibles de alginato de sodio y A. vera (100:0, 75:25, 50:50 y 25:75) sobre la pérdida de peso, color, firmeza, $\mathrm{pH}$ y acidez titulable de fresas, durante almacenamiento refrigerado $(0,3$, 9 y 12 días). Adicionalmente, se determinó el espesor y la opacidad de las películas obtenidas a partir de las soluciones formadoras de recubrimientos, utilizando el método de "casting". Los resultados mostraron que la combinación de $A$. vera y alginato de sodio tiene un efecto significativo en la reducción de pérdida de calidad de fresa, durante el almacenamiento en refrigeración. Todos los tratamientos con recubrimientos comestibles presentaron, al final del almacenamiento, menor pérdida de peso (7-16\%), mayor firmeza (entre 1,3 a 2,1 veces más), mayor retención del color y mayor acidez titulable que las muestras sin recubrimiento (control). El espesor de las películas disminuyó con el incremento de la adición de Aloe vera y la opacidad disminuyó significativamente con la adición de la sábila en $25 \%$ y $50 \%$ en las mezclas con alginato de sodio.

Palabras clave: fresa; Fragaria x ananassa; calidad poscosecha; recubrimientos comestibles; frutas mínimamente procesados.

\section{ABSTRACT}

Strawberries are highly perishable fruits, being susceptible to mechanical injury, decay and physiological disorders during storage. Applications of edible coatings have been shown to be promising 
as a tool to improve the quality and extend storage. The aim of this study was to evaluate the use of Aloe vera and sodium alginate as an edible coating in postharvest quality of cold storage strawberries. The composition of edible coatings of sodium alginate and $A$. vera (100: $0,75: 25,50: 50$ and 25:75) was studied on weight loss, color, firmness, $\mathrm{pH}$ and titrable acidity of strawberries during refrigerated storage $(0,3,9$ and 12 days). The thickness and opacity of the films were also determined, from the coating forming solutions using the casting method. The short shelf life and the high deterioration of the quality characteristics of strawberries during postharvest, justify to investigate alternative conservation techniques such as edible coatings, in order to reduce food loss and improve consumer acceptance The results showed that the combination of Aloe vera and sodium alginate has a significant effect in the reduction of the loss of the quality of the fruit during refrigerated storage. All coating treatments showed at the end of storage less weigth loss (7$16 \%$ ), greater firmness, (between 1,3 to 2,1 more times) more color retention and greater titrable acidity tan uncoated samples (Control). The addition of Aloe vera decreased the thickness of the films and the opacity decreased significantly with the addition of Aloe vera in 25 and 50\% in the mixtures with sodium Alginate.

Keywords: strawberry; Fragaria $\mathrm{x}$ ananassa; postharvest quality; edible coatings; minimally processed fruits.

\section{INTRODUCCIÓN}

La fresa (Fragaria x ananassa) es una fruta rica en sustancias relacionadas con beneficios para la salud, como vitamina $\mathrm{C}$, vitamina E, compuestos fenólicos y antocianinas (Dong et al. 2015); sin embargo, es altamente perecedera, lo cual, resulta en una corta vida poscosecha, debido a daños mecánicos, deterioro fisiológico, pérdida de agua, enfermedades fúngicas y alta tasa de respiración (Sogvar et al. 2016; Tahir et al. 2018). Colombia, en el 2016, tuvo una pérdida poscosecha en frutas y verduras de 1.165.219 toneladas, correspondiente al 60\% de las pérdidas totales de alimentos en el país (DNP, 2016). En países tropicales, como Colombia, la pérdida de fresa en manejo poscosecha puede llegar a ser hasta del 50\%. Cuando se mantienen a temperatura ambiente, los frutos se pueden deteriorar hasta en un $80 \%$, en un periodo de 8 horas (BarrazuelaRojas et al. 2017. En la actualidad, se encuentran en investigación métodos alternativos para prolongar su vida útil, como las películas o recubrimientos comestibles. Estos materiales protectores han sido de interés por investigadores y por la industria alimentaria en la última década, debido a su potencial para mejorar la inocuidad, la calidad y la funcionalidad de los productos alimenticios (Mellinas et al. 2016).

Diferentes biopolímeros, como proteínas, lípidos, polisacáridos o sus combinaciones pueden ser empleados para la elaboración de películas y recubrimientos con estructura cohesiva, para controlar el intercambio de gases, permeación de humedad o los procesos oxidativos, así como de reducir el crecimiento microbiano en los alimentos y obtener estabilidad e inocuidad de los productos (Valdés et al. 2017). También, se emplean como matrices para la inclusión de aditivos y de nutrientes, que pueden ser liberados de forma controlada en los alimentos, formando la base para sistemas de empaque inteligentes (Campos et al. 2011a).

El mucilago o gel de la hoja de Aloe vera consiste de, aproximadamente, 99,5\% agua y $0,5 \%$ de material sólido, que incluye polisacáridos, vitaminas, minerales y compuestos fenólicos (Benítez et al. 2015); adicional a esto, se ha estudiado su potencial como tratamiento para mantener la calidad poscosecha de frutas, previniendo la pérdida de humedad y de firmeza, así como controlando la tasa de respiración (Lin \& Zhao, 2007). Por otra parte, el alginato de sodio es un polisacárido lineal derivado del ácido alginico, el cual, es extraído de diferentes especies de algas marrones, como Laminaria hyperborea, Ascophyllum nodosum y Macrocystis pyrifera. Este es de interés para la industria de alimentos, debido a sus propiedades coloidales únicas, como formación de películas, biodegradibilidad y bioadhesividad (Pereira et al. 2011). Debido a esto, se ha estudiado su eficacia como tratamiento poscosecha, para mantener la calidad de frutas, durante el almacenamiento. Zhu et al. (2019) encontraron que el recubrimiento alginato de sodio aplicado a setas comestibles (Pholiota nameko) extiende su vida útil.

El objetivo de este trabajo fue estudiar el efecto de recubrimientos comestibles de sábila (A. vera) y alginato de sodio sobre parámetros de calidad de fresas, durante el almacenamiento refrigerado.

\section{MATERIALES Y MÉTODOS}

Frutos de fresa: Las fresas (Fragaria $\mathrm{x}$ ananassa), se adquirieron en una distribuidora local especializada en frutas. Se seleccionaron, considerando uniformidad en tamaño, forma y color, al menos, $80 \%$ de la superficie del fruto presentó una coloración rojiza, sin daños mecánicos o signos de enfermedad. La humedad del fruto, se determinó mediante secado con horno (Binder, Alemania), a $70^{\circ} \mathrm{C}$ por 24h. Los sólidos solubles, se determinaron por refractometría (Atago, Japón).

Extracción y acondicionamiento del gel de $A$. vera: Se seleccionaron hojas de sábila sanas y sin daños físicos para la extracción del gel. Estas presentaron una longitud de $64 \pm 4 \mathrm{~cm}$, un ancho de 11,7 $\pm 0,7 \mathrm{~cm}$, un grosor de $2,1 \pm 0,2 \mathrm{~cm}$ y un peso de 646 $\pm 62 \mathrm{~g}$, correspondiente a una madurez de 3 años, de acuerdo con lo reportado por Ahlawat et al. (2013). El gel, se retiró de la corteza externa de la hoja y posteriormente se homogenizó, mediante un mezclador. Con el fin de retirar fibras y material vegetal que pudiera estar presente, se centrifugó el material a 4000rpm, durante 10min, en una centrífuga (International Equipment Company, IEC HNSII, USA).

Elaboración de recubrimientos comestibles: Se elaboró una solución de alginato de sodio en agua destilada al 1,5\% (p/p), agitando durante una hora, a 800rpm, en un agitador mecánico (IKA RW 20 Digital, USA).

Para todas las soluciones formadoras, se utilizó $0,5 \%(\mathrm{p} / \mathrm{p})$ de glicerol, como plastificante y el 99,5\% restante, en una combinación 
de la solución de alginato de sodio (AS) y $A$. vera (AV), en las proporciones: 100:0, 75:25, 50:50, y 25:75, respectivamente. La mezcla de los componentes, se realizó a $600 \mathrm{rpm}$, durante $15 \mathrm{~min}$.

Elaboración de películas comestibles: Las soluciones de recubrimiento, se emplearon en las fresas y en la elaboración de películas, por el método de moldeado por evaporación del solvente ("solvent-casting"). Para formar la película, se depositaron $20 \mathrm{~mL}$ de solución formadora, en una caja Petri de $90 \mathrm{~mm}$ de diámetro. Posteriormente, se secó a $40^{\circ} \mathrm{C}$, durante $48 \mathrm{~h}$, en un horno de convección natural (Binder, Alemania).

Aplicación del recubrimiento comestible en fresas: Los frutos, se sumergieron en la solución formadora durante 30 s y, posteriormente, se secaron a $20^{\circ} \mathrm{C}$, durante $2 \mathrm{~h}$. Los frutos recubiertos, se ubicaron en cajas plásticas ( 6 fresas por caja) que, después, se llevaron a refrigeración a $4 \pm 0,5^{\circ} \mathrm{C}$. Las características de calidad de los frutos fueron analizadas al inicio de la prueba (día 0) y a los 3, 9 y 12 días de almacenamiento. Los experimentos, se realizaron por triplicado.

\section{Determinación de las características de calidad de los frutos de fresa:}

Pérdida de peso: El peso de la fruta, se registró en el día inicial del tratamiento (día 0) y a los 3, 9 y 12 días. La pérdida de peso (PP), se expresó como porcentaje.

Color: Se midieron los parámetros $\mathrm{L}^{*}, \mathrm{a}^{*}$ y b* del espacio de color CIELAB en las fresas, empleando un colorímetro (Color Flex Hunterlab, USA). A partir de esta información, se calculó el croma (C) o pureza de color, durante el almacenamiento, empleando la ecuación 1.

$$
C=\sqrt{a^{2}+b^{2}}
$$

ecuación 1

Firmeza: Se evaluó usando un texturometo EZ test (Shimadzu, USA) y un punzón de $50 \mathrm{~mm}$ de longitud y $3 \mathrm{~mm}$ de diámetro. La fruta, se penetró hasta $6 \mathrm{~mm}$ de profundidad, a una velocidad de $40 \mathrm{~mm}$ / min. Los resultados fueron expresados como la fuerza máxima en Newton $(\mathrm{N})$, requerida para lograr la penetración del fruto.

Sólidos solubles, pH y acidez titulable: Para la medición de estas características, se extrajo el jugo de las fresas, empleando un exprimidor manual. Los sólidos solubles iniciales de cada fruta fueron determinados con un refractómetro digital Atago RX-7000 (Atago, Japón). El pH fue determinado a partir de la mezcla del jugo obtenido de seis fresas, con un medidor de $\mathrm{pH}$ Orion Modelo 520 A (Thermo Ficher Scientific, USA). La acidez titulable, se determinó titulando con $\mathrm{NaOH} 0,1 \mathrm{~N}$ una alícuota de $5 \mathrm{~mL}$ de jugo de fresa mezclada con $95 \mathrm{~mL}$ de agua destilada hasta un $\mathrm{pH}$ de 8,1. El porcentaje de acidez titulable (\%AT) como ácido cítrico en el fruto, se calculó a partir de la ecuación 2.

$$
\% \mathrm{AT}=\frac{\mathrm{V}(\mathrm{NaOH}) 0,10,064}{\mathrm{~m}_{\text {alicuota }}} \times 100
$$

ecuación 2
Donde, $\mathrm{V}(\mathrm{NaOH})$ son los mililitros de $\mathrm{NaOH}$ empleados para la titulación; 0,1 es la normalidad de la solución de $\mathrm{NaOH}$; 0,064 es el factor de conversión para el ácido cítrico y m es la cantidad de muestra empleada para el análisis.

\section{Caracterización de las películas:}

Espesor: El espesor de las películas, se midió utilizando un micrómetro digital (IGAGING, USA). Se realizaron 5 mediciones en diferentes lados de la muestra.

Opacidad: La opacidad (O) fue determinada usando los valores de luminosidad de las películas contra un fondo estándar blanco ( $L^{*}$ blanco) y un fondo estándar negro $\left(L^{*}\right.$ negro), donde un valor de $100 \%$ indica una película opaca y un valor de $0 \%$, una película transparente. Se empleó la ecuación 3 para calcular esta característica.

$\% 0=\frac{\mathrm{L}_{\text {Negro }}}{\mathrm{L}_{\text {Blanco }}} \times 100$

ecuación 3

Análisis estadístico: Se realizó un análisis de varianza (ANOVA) con un nivel de confianza del 95\%, para determinar la significancia de los tratamientos sobre las características de calidad de la fresa. El procesamiento de los datos, se realizó con el software estadístico Minitab 18. Para las diferencias significativas entre tratamientos, se efectuó una prueba Post-ANOVA (Método de comparación en parejas de Tukey).

\section{RESULTADOS Y DISCUSIÓN}

Los frutos de fresa presentaron un contenido de humedad de 92,1士 $0,1 \%$ y un contenido de sólidos solubles $6.8 \pm 0.2^{\circ} \mathrm{Brix}$. El gel de Aloe vera presentó un contenido de humedad de 99,17 $\pm 0,08 \%$, solidos solubles de $0,6 \pm 0,1^{\circ}$ Brix y un $\mathrm{pH}$ de $4,51 \pm 0,04$

Pérdida de peso: La pérdida de peso es un índice de calidad usado ampliamente en la poscosecha de frutas. En la figura 1, se observa que las fresas en todos los tratamientos perdieron significativamente peso durante el almacenamiento $(\mathrm{p}<0,05)$. Este comportamiento está asociado a la alta tasa de respiración del fruto, lo que lleva a la evaporación de agua en la superficie, ocasionando la pérdida de peso (Campos et al. 2011b). Se observó también, que todos los tratamientos recubiertos con películas comestibles mostraron significativamente $(\mathrm{p}<0,05)$ menores pérdidas de peso a los 9 y 12 días de almacenamiento respecto a la muestra control, que alcanzó las mayores pérdidas, 10,39 y 20,08\%, respectivamente. Este resultado indica que los recubrimientos comestibles brindaron protección a la fruta frente a la pérdida de humedad, logrando retardar la deshidratación. Comportamiento similar fue reportado por Olivas et al. (2007) en recubrimientos con alginato de calcio en rodajas de manzana, donde las muestras sin recubrimiento presentaron pérdidas de $20 \%$, mientras que las manzanas recubiertas solo del 7\%. De igual manera, Sogvar et al. (2016) encontraron pérdidas del $7 \%$ en peso para fresas recubiertas con Aloe vera, durante un almacenamiento refrigerado de 9 días. Por otro lado, las combinaciones de alginato de sodio y $A$. vera lograron reducir la migración de humedad desde la fruta, mediante la creación de una barrera física entre el alimento y el medio ambiente; cuanto mayor 
porcentaje fue el alginato y menor el $A$. vera, menor fue la pérdida de peso. Este comportamiento puede estar asociado al mayor espesor de película, ya que, a menor cantidad de $A$. vera y mayor alginato de sodio, mayor es el espesor del recubrimiento (Tabla 1). La prueba post-Anova mostró que las medias de estos tratamientos fueron estadísticamente diferentes del control.
Color en términos de claridad y pureza de color: Los valores de L* (claridad) de las muestras control en el día cero fue de 32,2 $\pm 0,4$, mientras que las fresas recubiertas con las distintas mezclas de alginato de sodio y $A$. vera presentaron valores ligeramente superiores (mayor claridad), excepto el recubrimiento $100 \%$ de alginato $(30,4 \pm 0,9)$. Al final del almacenamiento (día 12), todos los

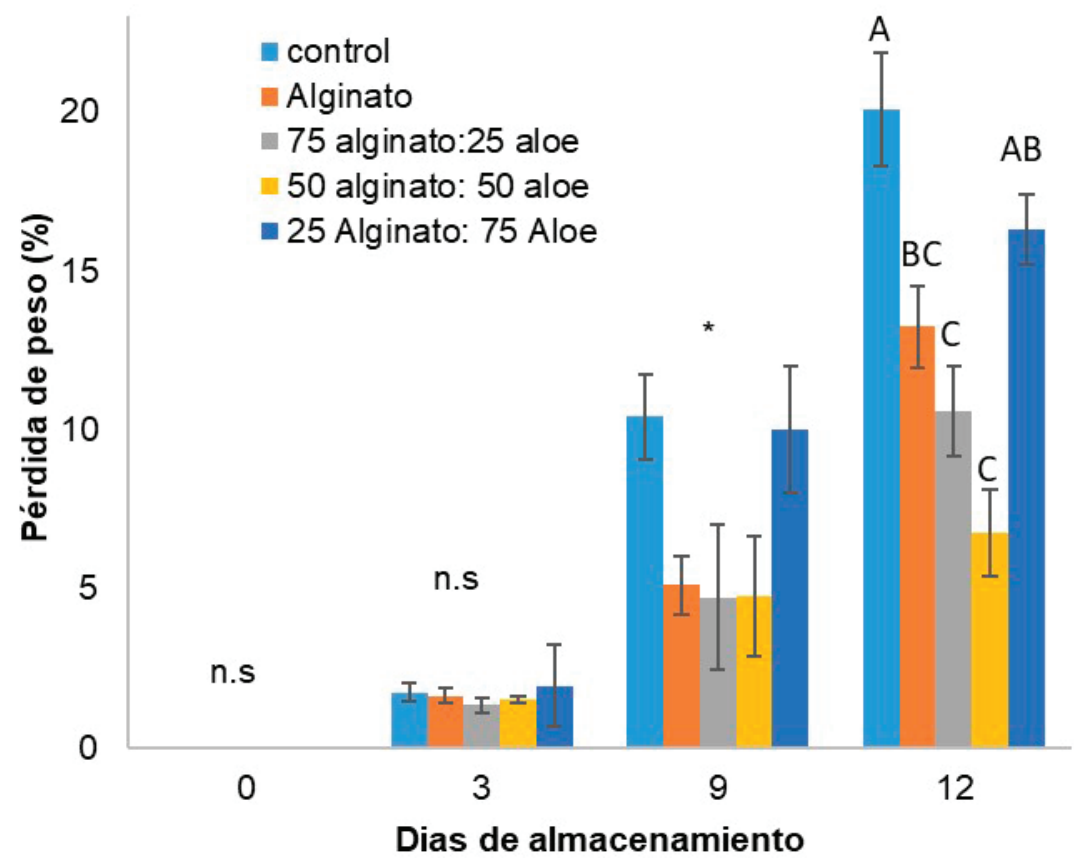

Figura 1. Pérdida de peso en frutos de fresa con y sin recubrimientos de Alginato-Aloe vera. n.s indica que no hay diferencia significativa; $*$ indica diferencia estadísticamente significativa $(\mathrm{p}<0,05)$. Las medias que no comparten una letra son significativamente diferentes.

tratamientos disminuyeron $\mathrm{L}^{*}$ significativamente $(\mathrm{p}<0,05)$, asociado a pérdida de claridad (ligero oscurecimiento). La muestra control disminuyó hasta $18,9 \pm 1,4$ y los tratamientos con recubrimiento presentaron valores inferiores, entre $17 \pm 1,4$ y $14,2 \pm 1,4$, lo que significa disminución de claridad, por efecto del tiempo de almacenamiento. El post-Anova a los 12 días mostró que las medias de los tratamientos fueron estadísticamente diferentes, siendo el tratamiento de alginato de sodio el de mayor pérdida de claridad, con 14,2 $\pm 1,4$ (mayor oscurecimiento). Este resultado puede ser debido a la mayor opacidad de la película de alginato de sodio 100\% (Tabla 1), mientras que los dos tratamientos de mezcla alginato- $A$. vera presentaron menor pérdida de claridad, indicando una sinergia en estos dos componentes.

La figura 2 muestra que todos los tratamientos indicaron una disminución significativa $(\mathrm{p}<0,05)$ de la pureza de color $\left(\mathrm{C}^{*}\right)$, por efecto del tiempo de almacenamiento, lo que sugiere que la fresas presentaron menor intensidad de color rojo. Similares resultados expusieron Perdones et al. (2012) en el almacenamiento de fresas. El análisis estadístico permitió encontrar diferencias significativas entre las medias de los tratamientos en el día 3 y 12. En el día 12, la muestra control presentó mayor pureza de color que el tratamiento
$100 \%$ de alginato de sodio, pero inferior a las tratadas con mezcla alginato- $A$. vera. El menor valor de $C^{*}$ en las muestras tratadas con alginato de sodio también fue observado por Guerreiro et al. (2015), Velickova et al. (2013) y Perdones et al. (2012). Es evidente que el recubrimiento con $A$. vera potenció el $C^{*}$ respecto a la muestra control y la de alginato de sodio; comportamiento similar, se reportan en estudios en kiwi (Benítez et al. 2015) y en pomelo y duraznos (Guillén et al. 2013).

Firmeza: La figura 3 evidencia que en todos los tratamientos la firmeza disminuyó significativamente durante el tiempo de almacenamiento $(\mathrm{p}<0,05)$, atribuida a la degradación de la laminilla media de la pared celular en las células del parénquima cortical de la fresa (Sogvar et al. 2016). En todos los casos, las muestras tratadas con recubrimientos comestibles presentaron significativamente $(\mathrm{p}<0,05)$ mayor firmeza que la muestra control, siendo las muestras recubiertas con alginato de sodio las que mostraron significativamente $(p<0,05)$ mayor firmeza hasta el día 12. Se ha evidenciado que los recubrimientos disminuyen el ablandamiento del producto; esto se debe, a que actúan como una barrera para el oxígeno, especialmente, aquellos a base de hidrocoloides, como los alginatos, reduciendo la actividad metabólica y, con ello, los procesos 
Tabla 1. Espesor y opacidad de películas de Alginato-A.vera. Letras diferentes indican diferencias estadísticamente significativas.

\begin{tabular}{|c|c|c|}
\hline Composición & Espesor $(\mathbf{m m})$ & O (\%) \\
\hline Alginato & $0,110 \pm 0,005^{\mathrm{a}}$ & $32 \pm 3^{\mathrm{a}}$ \\
\hline 75 Alginato: 25 Aloe & $0,10 \pm 0,01^{\mathrm{ab}}$ & $23 \pm 4^{\mathrm{b}}$ \\
\hline 50 Alginato:50 Aloe & $0,08 \pm 0,01^{\mathrm{bc}}$ & $23 \pm 1^{\mathrm{b}}$ \\
\hline 25 Alginato: 75 Aloe & $0,054 \pm 0,002^{\mathrm{c}}$ & $29 \pm 1^{\mathrm{ab}}$ \\
\hline
\end{tabular}

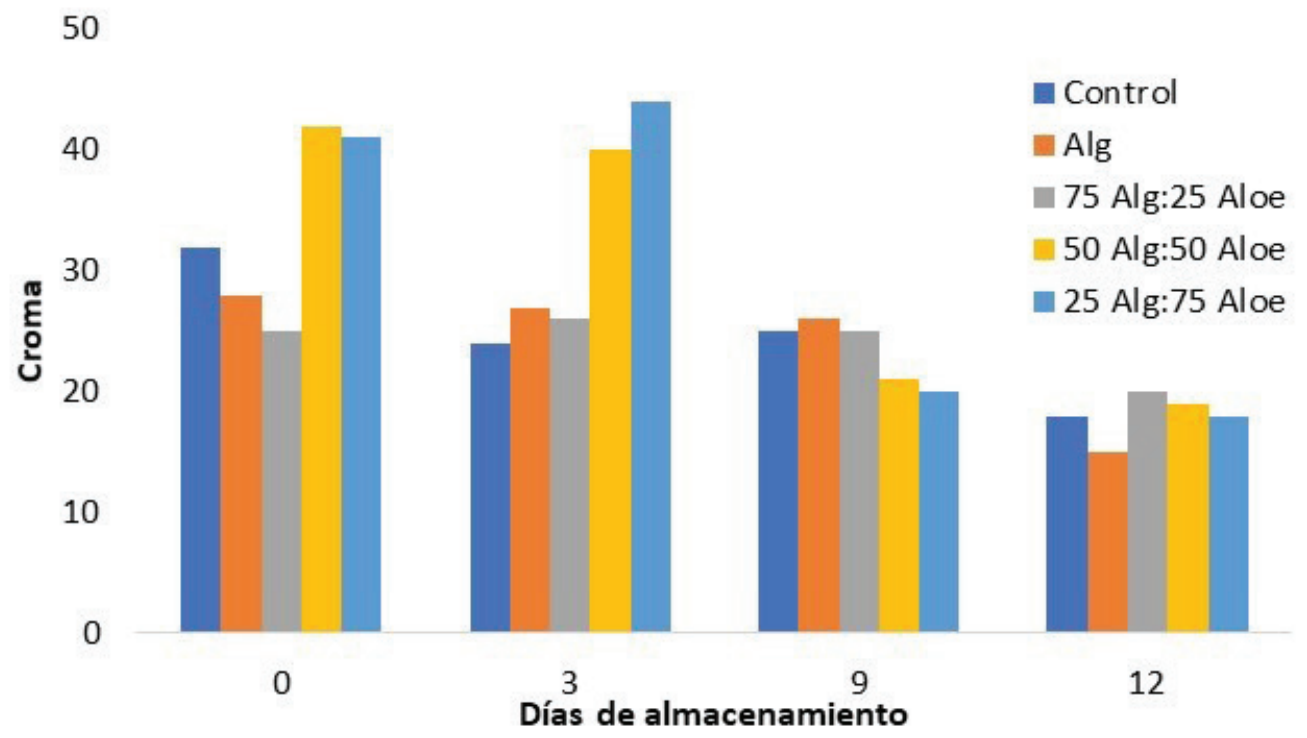

Figura 2. Croma en frutos de fresa con y sin recubrimientos de Alginato-Aloe vera. n.s indica que no hay diferencia significativa, mientras que * indica diferencia estadísticamente significativa $(\mathrm{p}<0,05)$.

asociados a la pérdida de firmeza (De Ancos et al. 2015; Sogvar et al. 2016). Guerreiro et al. (2015) encontraron que fresas recubiertas con alginato presentaron mayor firmeza durante el almacenamiento respecto a otras recubiertas con diferentes tipos de polisacáridos. Por otro lado, la mayor firmeza encontrada en la fruta recubierta con alginato puede estar asociada al espesor de la película (Tabla 1). Otro de los factores a considerar es la permeabilidad de las diferentes mezclas de alginato y aloe. Se ha reportado que el Aloe vera presenta una alta permeabilidad al vapor de agua, con valores cercanos a los $47 \mathrm{~g} \mathrm{~mm} / \mathrm{d} \mathrm{m}^{2} \mathrm{~Pa}$. La adición de un hidrocoloide (como la goma gelano), disminuye la permeabilidad de las películas de aloe, pasando de 47 a $21 \mathrm{~g} \mathrm{~mm} / \mathrm{d} \mathrm{m}^{2} \mathrm{~Pa}$, disminuyendo la salida de agua del fruto al medio y, por consiguiente, explicando la mayor firmeza del fruto por acción del alginato (Alvarado et al. 2012).

pH y acidez titulable: La figura 4 a presenta valores de $\mathrm{pH}$ y la figura 4b la acidez titulable en frutos de fresa con y sin recubrimiento. Se observaron diferencias significativas $(p<0,05)$ entre el pH de las muestras, a los 3, 9 y 12 días de almacenamiento. $\mathrm{El} \mathrm{pH}$, se incrementó en todos los tratamientos durante el tiempo de almacenamiento, notándose mayor $\mathrm{pH}$ en la muestra control, a los 9 y 12 días. El incremento en el pH de las muestras sin recubrimiento puede estar asociado al consumo o rompimiento de ácidos orgánicos durante el almacenamiento de la fresa, los cuales, son empleados durante la respiración u otros procesos metabólicos. Comportamiento similar, se registró en estudios de recubrimientos de fresa (Sogvar et al. 2016) y de tomate (Athmaselvi et al. 2013).

Respecto a la acidez titulable, se hallaron diferencias significativas $(\mathrm{p}<0,05)$ entre los tratamientos a los 3, 6 y 9 días de almacenamiento. Todos los tratamientos mostraron disminución de la acidez titulable durante el almacenamiento. Este descenso de la acidez es atribuido al consumo de ácidos orgánicos, siendo predominante en fresas el ácido cítrico (Famiani et al. 2015), por parte de los procesos metabólicos, durante la respiración de la fruta (Sogvar et al. 2016). No se evidencia una tendencia de la relación de concentración del recubrimiento sobre la acidez titulable; sin embargo, todos los tratamientos con recubrimiento presentaron mayor porcentaje de acidez titulable que la muestra control, comportamiento que puede ser atribuido a que los recubrimientos de alginato y de $A$. Vera reducen el ingreso de oxígeno requerido para los procesos de respiración en el fruto, protegiendo la reducción de los ácidos orgánicos, principalmente, el ácido cítrico de la fresa. 


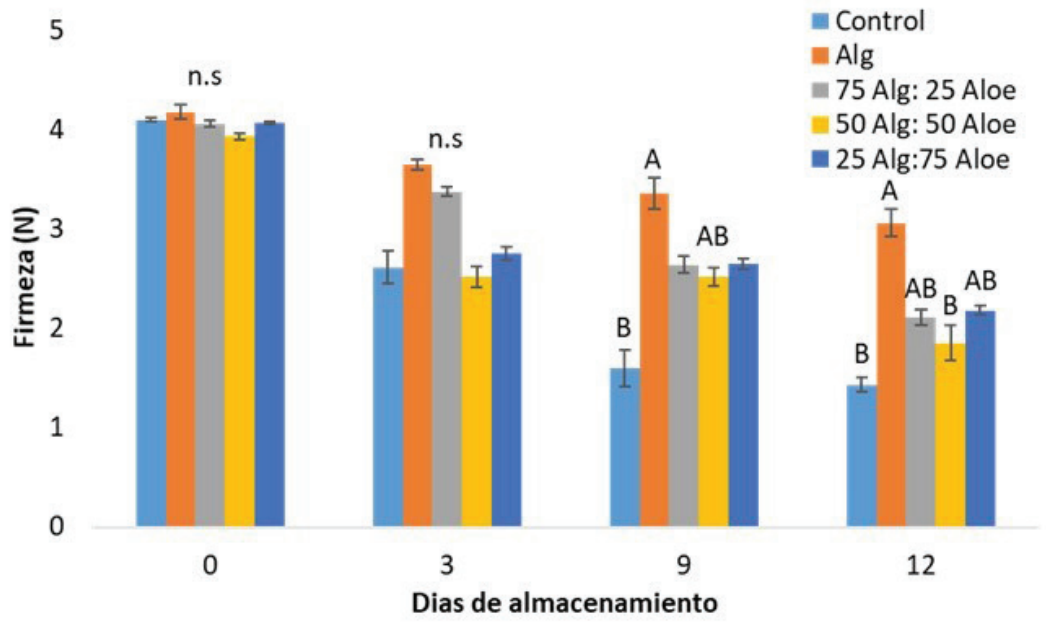

Figura 3. Firmeza en frutos de fresa con y sin recubrimientos de Alginato- $A$. vera. n.s indica que no hay diferencia significativa. Las medias que no comparten una letra son significativamente diferentes.

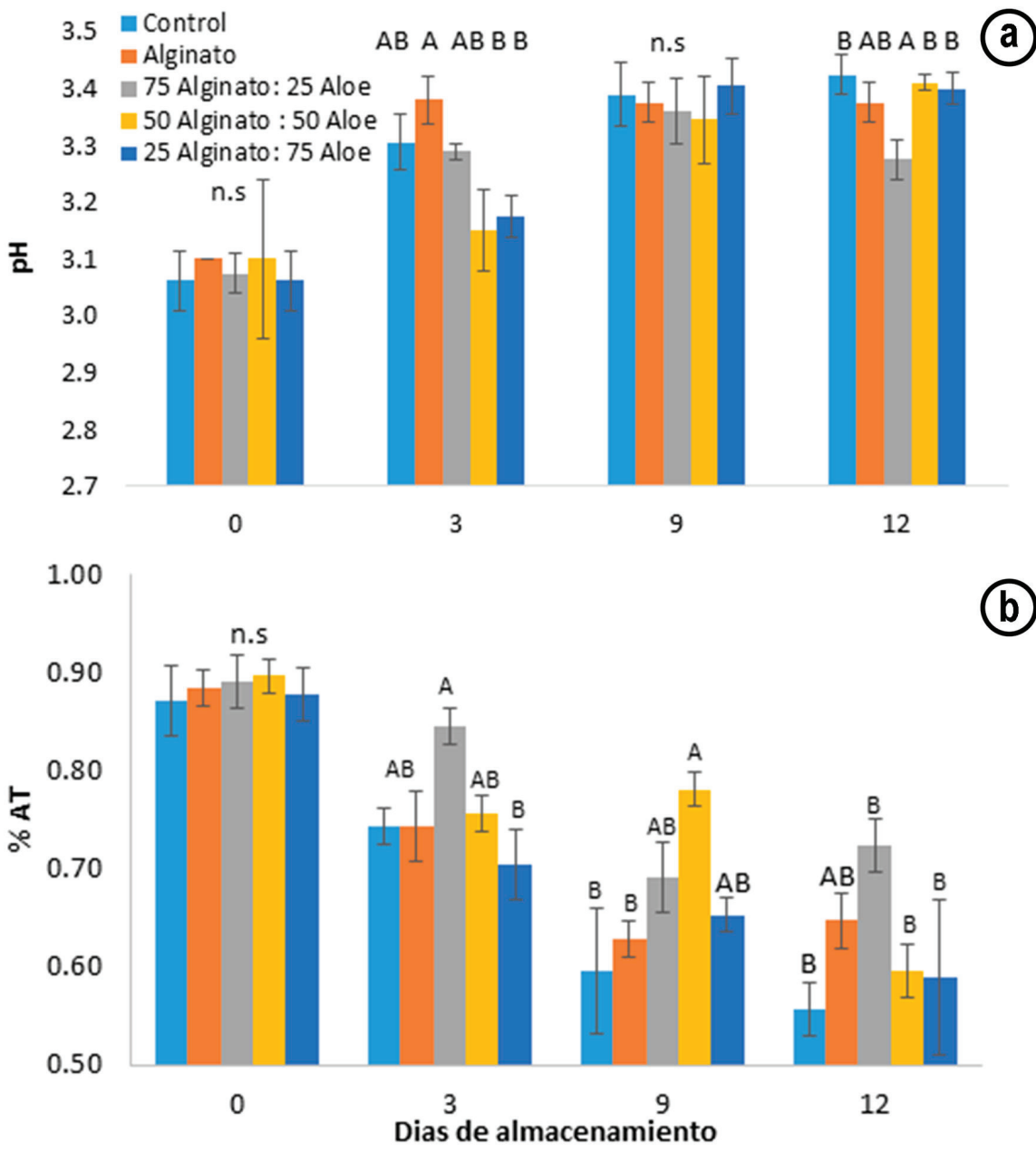

Figura 4. pH. a) y acidez titulable, b) en frutos de fresa con y sin recubrimiento. n.s indica que no hay diferencia significativa. Las medias que no comparten una letra son significativamente diferentes. 


\section{Caracterización de las películas:}

Espesor: El análisis estadístico mostró diferencias significativas $(p<0,05)$ entre el espesor de las películas en los diferentes tratamientos. La película de alginato de sodio fue la que presentó mayor espesor. Se observó que la adición de $A$. vera a la mezcla reduce el grosor de las películas obtenidas (Tabla 1). Este comportamiento también fue reportado por Pereira et al. (2011), en la elaboración de películas de alginato- $A$. vera. El alginato de sodio es un hidrocoloide que liga el agua dentro de la película; una reducción de su porcentaje en la mezcla permitirá una salida más fácil del agua durante el secado y, por consiguiente, una disminución en el espesor. El espesor de la película es un factor importante en el intercambio de agua y de gases con el ambiente. Un incremento en el grosor de la película reducirá la tasa de difusión (Pavlath \& Orts, 2009), lo que podría explicar los resultados encontrados para la pérdida de peso en la combinación 25 alg: 75 Aloe, donde el menor espesor de película $(0,054 \pm 0,002 \mathrm{~mm})$ presentó la mayor pérdida de peso entre los recubrimientos $(16,31 \%$ para el día 12 de almacenamiento).

Opacidad: Se encontraron diferencias significativas $(p<0,05)$ de la opacidad entre los tratamientos (Tabla 1). La película de alginato de sodio presentó la mayor opacidad en las películas, con $32 \pm 3 \%$; sin embargo, en la adición de $A$. vera en la mezcla con alginato de sodio en las proporciones de 25 y $50 \%$ disminuyó significativamente $(\mathrm{p}<0,05)$ la opacidad respecto a las películas de $100 \%$ de alginato de sodio. La opacidad es una propiedad crítica cuando la película se emplea sobre la superficie de un alimento. Este es un indicador de cuánta luz puede pasar a través del material. Las diferencias en la opacidad de las películas pueden ser explicadas por la combinación de sustancias con índices de refracción distintos, así como a cambios en la estructura interna durante el secado de la película, de acuerdo con lo expuesto por Fabra et al. (2009).

El uso de recubrimientos comestibles de mezclas de Aloe de vera y de alginato de sodio en frutos de fresa, evidencia mayor retención de parámetros de calidad (menor pérdida de peso, mayor firmeza, mayor retención del color y mayor acidez titulable), comparado con muestras sin recubrimiento. Se evidencia que cuanto menor es la concentración de Aloe vera en la mezcla del recubrimiento, menor es la pérdida de calidad del fruto, lo que indica la sinergia entre el Aloe vera y Alginato de sodio en la aplicación de recubrimientos comestibles en la conservación poscosecha de la fresa.

Se recomienda continuar investigando sobre los efectos de la composición del recubrimiento (relación alginato-aloe) en la aceptación sensorial del producto, la retención de compuestos funcionales, como antioxidantes (antocianinas y fenoles), así como una evaluación económica del costo-beneficio de la aplicación del recubrimiento.

Conflicto de interés: El manuscrito fue preparado y revisado con participación de todos los autores, quienes declaramos que no existe conflicto de intereses que ponga en riesgo la validez de los resultados presentados. Financiación: Esta investigación fue financiada por el Programa Jóvenes Investigadores e innovadores, convocatoria $\mathrm{N}^{\circ}$ 761-2016 COLCIENCIAS.

\section{REFERENCIAS}

1. AHLAWAT, K.S.; AHLAWAT, S.; GULIA, N.; KHATKARI, B.S. 2013. Effect of plant maturity on leaf growth, yield and physicochemical properties of aloe vera gel. AgroFOOD industry hi-tech. (Italy). 24(1):22-24.

2. ALVARADO-GONZÁLEZ, J.S.; CHANONA-PÉREZ, J.J.; WELTI-CHANES, J.S.; CALDERÓN-DOMÍNGUEZ, G.; ARZATE-VÁZQUEZ, I.; PACHECO-ALCALÁ, S.U.; GARIBAY-FEBLES, V.; GUTIÉRREZ-LÓPEZ, G.F. 2012. Optical, microstructural, functional and nanomechanical properties of Aloe vera gel/gellan gum edible films. Rev. Mexicana de ingeniería química. (México). 11(2):193-210.

3. ATHMASELVI, K.; SUMITHA, P.; BASKARAN, R. 2013. Development of Aloe vera based edible coating for tomato. International Agrophysics. (Poland). 27(4):369375. https://doi.org/10.2478/intag-2013-0006

4. BARRAZUELA-ROJAS, S.G.; FALCONI, J.F.; NAVARRO OJEDA, M.N.; OLEAS-LOPEZ, J.M.; MENDOZAZURITA, G.X. 2017. Pysicochemical properties and application of edible coatings in strawberry (Fragaria $\mathrm{x}$ ananassa) preservation. Rev. Fac. Nac. Agron. (Colombia). 71(3):8631-8641. https.//doi.org/10.15446/rfnam. v71n3.73548

5. BENÍTEZ, S.; ACHAERANDIO, I.; PUJOLA, M.; SEPULCRE, F. 2015. Aloe vera as an alternative to traditional edible coatings used in fresh cut fruits: A case of study with kiwifruit slice. LWT- Food Science and Technology. (USA). 61(1):184-193. https://doi.org/10.1016/j.lwt.2014.11.036

6. CAMPOS, C.A.; GERSCHENSON, L.N.; FLORES, S.K. 2011a. Development of edible films and coatings with antimicrobial activity. Food Bioprocess Technol. (USA). 4(6):849-875. https://doi.org/10.1007/s11947-010-0434-1

7. CAMPOS, R.P.; KWIATKOWSKI, A.; CLEMENTE, E. 2011b. Post-harvest conservation of organic strawberries coated with cassava starch and chitosan. Rev. Ceres. (Brazil). 58(5):554-560. https://doi.org/10.1590/S0034737X2011000500004

8. DE ANCOS, B.; GONZÁLEZ-PEÑA, D.; COLINA-COCA, C.; SÁNCHEZ-MORENO, C. 2015. Uso de los productos de IV y V gama. Rev. Iberoamericana de Tecnología Postcosecha. (México). 16(1):8-17.

9. DEPARTAMENTO NACIONAL DE PLANEACIÓN, DNP. 2016. Pérdida y desperdicio de alimentos en Colombia. Estudio de la dirección de seguimiento y evaluación de políticas públicas. Disponible desde internet en: https:// colaboracion.dnp.gov.co/CDT/Prensa/Publicaciones/ 
P $\%$ C 3\%A9rdida $\% 20 y \% 20$ desperdicio $\% 20$ de $\% 20$ alimentos $\% 20$ en $\% 20$ colombia.pdf [con acceso el $17 / 05 / 2018]$

10. DONG, F.; SHUJUN, L.; ZHONGMING, L.; ZHU, K.; WANG, X.; JIN, C. 2015. Improvement of quality and shelf life of strawberry with nanocellulose/chitosan composite coatings. Bangladesh J. Botany. (BANGLADÉS). 44(5):709717.

11. FAMIANI, F; BATTISTELLI, A.; MOSCATELLO, S.; CRUZCASTILLO, J.G.; WALKER, R.P. 2015. The organic acids that are accumulated in the flesh of fruits: occurrence, metabolism and factors affecting their contents-a review. Rev. Chapingo Serie Horticultura. (México) 21(2):97-128. https://doi.org/10.5154/r.rchsh.2015.01.004

12. FABRA, M.J.; TALENS, P.; CHIRALT, A. 2009. Microstructure and optical properties of sodium caseinate films containing oleic acid-beeswax mixtures. Food Hydrocolloids. (Netherlands). 23(3):676-683. https://doi.org/10.1016/j. foodhyd.2008.04.015

13. GUERrEiro, A.; GAGO, C.M.L.; FALEIRO, M.L.; MIGUEL, M.G.C.; ANTUNES, M.D.C. 2015. The use of polysaccharide-based edible coatings enriched with essential oils to improve shelf-life of strawberries. Postharvest Biology and Technology. 110:51-60. https:/ / doi.org/10.1016/j.postharvbio.2015.06.019

14. GUILLÉN, F.; DÍAZ-MULA, H.; ZAPATA, P.; VALERO, D.; SERRANO, M.; CASTILLO, S.; MARTÍNEZ-ROMERO, D. 2013. Aloe arborescens and Aloe vera gels as coatings in delaying postharvest ripening in peach and plum fruit. Postharvest Biology and Technology. 83:54-57. https:// doi.org/10.1016/j.postharvbio.2013.03.011

15. LIN, D.; ZHAO, Y. 2007. Innovations in the development and application of edible coatings for fresh and minimally processed fruits and vegetables. Comprehensive reviews in Food Sci. Food Safety. (USA) 6(3):59-75. https://doi. org/10.1111/j.1541-4337.2007.00018.x

16. MELLINAS, C.; VALDÉS, A.; RAMOS, M.; BURGOS, N.; GARRIGÓS, M.C.; JIMÉNEZ, A. 2016. Active edible films: Current state and future trends. J. Appl. Polym. Sci. (USA) 133(2). https://doi.org/10.1002/app.42631

17. OLIVAS, G.I.; MATTININSON, D.S.; BARBOSA-CÁNOVAS, G.V. 2007. Alginate coatings for preservation of minimally processed 'Gala' apples. Postharvest Biology and Technology. (Netherlands). 45(1):89-96. https://doi. org/10.1016/j.postharvbio.2006.11.018
18. PAVLATH, A.E.; ORTS, W. 2009. Edible films and coatings: Why, What and How? En: Embuscado, M.E., Huber, K.C. (eds). Edible Films and Coatings for Food Aplications. Ed. Springer (New York). 12p.

19. PERDONES, A.; SÁNCHEZ-GONZÁLEZ, L.; CHIRALT, A.; VARGAS, M. 2012. Effect of chitosan-lemon essential oil coatings on storage-keeping quality of strawberry. Postharvest Biology and Technology. 70:32-41. https:// doi.org/10.1016/j.postharvbio.2012.04.002

20. PEREIRA, R.; TOJEIRA, A.; VAZ, D.C.; MENDES, A.; BÁRTOLO, P. 2011. Preparation and Characterization of Films Based on Alginate and Aloe vera. Internal J. Polymer Analysis and Characterization. (United Kingdom). 16(7):449464. http:/ / doi.org/10.1080/1023666X.2011.599923

21. SOGVAR, O.B.; SABA, M.K.; EMAMIFAR, A. 2016. Aloe vera and ascorbic acid coatings maintain postharvest quality and reduce microbial load of strawberry fruit. Postharvest Biology and Technology. 114:29-35. https:/ / doi.org/10.1016/j.postharvbio.2015.11.019

22. TAHIR, H.E.; XIAOBO, Z.; JIYONG, S.; MAHUNU, G.K; ZHAI, X.; MARIOD, A.A. 2018. Quality and postharvestshelf life of cold-stored strawberry fruit as affected by gum arabic (Acacia senegal) edible coating Journal of Food Biochemistry. (USA). 42(3):1-10. https://doi.org/10.1111/ jfbc. 12527

23. VALDÉS, A.; RAMOS, M.; BELTRÁN, A.; JIMENEZ, A.; GARRIGÓS, M.C. 2017. State of the Art of Antimicrobial Edible Coatings for Food Packaging Applications. Coatings. (Switzerland) 7(4)56:1-23. https://doi.org/10.3390/ coatings 7040056

24. VELICKOVA, E.; WINKELHAUSEN, E.; KUZMANOVA, S.; ALVES, V.D.; MOLDÃO- MARTINS, M. 2013. Impact of chitosan-beeswax edible coatings on the quality of fresh strawberries (Fragaria ananassa cv Camarosa) under commercial storage conditions. LW'T-Food Science and Technology. (USA). 52(2):80-92. https://doi.org/10.1016/j. lwt.2013.02.004

25. ZHU, D.; GUO, R.; LI, W.; SONG, J.; CHENG, F. 2019. Improved postharvest preservation effects of Pholiota nameko mushroom by sodium alginate-based edible composite coating. Food and Bioprocess Technology. (USA). 12(4):587-598. https://doi.org/10.1007/s11947019-2235-5 